\title{
ÊTRE MOZAMBICAIN DANS LE MPUMALANGA
}

La province frontalière de l'Afrique du Sud avec le Mozambique abrite une importante minorité issue ou supposée originaire de ce pays. Particulièrement dans les municipalités orientales de Mbombela et Nkomazi, la présence de Mozambicains remonte à un peuplement ancien dû à leur emploi massif dans les mines et dans l'agriculture. Plusieurs migrations se sont superposées dans l'histoire récente, aboutissant à un enchevêtrement complexe de situations juridiques. Dans les années quatre-vingt-dix, avec simultanément la fin de la guerre civile au Mozambique et l'extinction de l'apartheid, des Mozambicains des anciens homelands se sont retrouvés sans papiers, des réfugiés ont été régularisés, d'autres ont été rapatriés de gré ou de force. Depuis lors, la migration est devenue un phénomène structurel, de même que les déportations. Les Mozambicains, relativement fondus dans la population, constituent, à cause de leur précarité statutaire, une main-d'œuvre recherchée dans certains secteurs économiques urbains et périurbains. En dépit de cette intégration de facto, ils ont à subir une ségrégation héritée de l'apartheid, ainsi que les discours stigmatisants associés à la figure du migrant abusif et délinquant. Incertaines et pragmatiques, alternant tolérance et répression, les politiques publiques à leur endroit sont un constant défi aux droits fondamentaux des personnes.

\section{Ser moçambicano no Mpumalanga}

A região fronteiriça da África do Sul com Moçambique abriga uma minoria importante oriunda ou supostamente originária deste país. Especialmente nos municípios orientais de Mbombela et Nkomazi, a presença de moçambicanos remonta a um povoamento antigo devido à sua contratação em massa nas minas e na agricultura. Várias migrações sobrepuseram-se na história recente, levando a um emaranhado complexo de situações jurídicas. Durante os anos 1990, com o fim da guerra civil em Moçambique simultâneo à extinção do apartheid, muitos moçambicanos dos antigos homelands encontraram-se sem documentos, muitos refugiados foram regularizados e outros foram repatriados a bem ou a mal. Desde então, a migração tornou-se un fenómeno estrutural, bem como as deportações. Os moçambicanos, relativamente misturados com a população, constituem, devido à sua precariedade estatutária, uma mão-de-obra procurada em certos sectores económicos urbanos e das periferias urbanas. Apesar desta integração de facto, eles são alvo de uma segregação herdada do apartheid, bem como os discursos estigmatizantes associados à figura do imigrante abusador e delinquente. Incertas e pragmáticas, alternando tolerância e repressão, as políticas públicas no seu lugar são um desafio constante aos direitos fundamentais das pessoas.

\section{Being Mozambican in Mpumalanga}

South Africa's border province with Mozambique is home to a large minority from Mozambique or assumed to be of Mozambican origin. In the eastern towns of Mbombela and Nkomazi, in particular, the presence of Mozambicans goes back a long way, due to their large-scale employment in the mines and in farming. Several waves of migration have followed each other in recent times, resulting in a complex tangle of legal statuses. In the 1990s, with the simultaneous 
end of civil war in Mozambique and the end of apartheid, some Mozambicans from the former homelands found themselves undocumented immigrants, some refugees had their status regularised, while others were repatriated voluntarily or by force. Since then, migration has become a structural phenomenon, as have deportations. Mozambicans are relatively mixed into the population and provide much sought-after labour in certain urban and suburban sectors, due to their precarious status. Despite this de facto integration, they do face segregation inherited from the apartheid system, as well as the stigmatising discourse associated with the figure of the illegal immigrant and delinquent. Public policies are uncertain and pragmatic, alternating tolerance and repression. Public policies on them are uncertain and pragmatic, alternating tolerance and repression, and are a constant challenge to the fundamental rights of people.

$\mathrm{L}$ ors des événements de mai 2008 en Afrique du Sud, les provinces du Limpopo det du Mpumalanga, respectivement frontalières du Zimbabwe au Nord et du Mozambique à l'Est furent presque entièrement épargnées par les émeutes xénophobes, en dépit de la forte proportion d'étrangers qui s'y rencontre. Dans la province du Mpumalanga (ancien Eastern Transvaal), dont la partie orientale, le Lowveld (bas-plateaux), se termine sur la route de Maputo par un étroit corridor entre le Parc Kruger et le royaume du Swaziland, vit une importante minorité de «Mozambicains » - on verra plus loin le sens de ces guillemets - parfois installés depuis très longtemps, et aujourd'hui disséminés dans les exploitations agricoles et dans toutes les formes d'habitat urbain et périurbain héritées de l'ère de l'apartheid. Disons par anticipation que cette population est très hétérogène à tout point de vue.

Quoique malaisément identifiables, surtout dans les districts proches de la frontière où les habitants noirs partagent un fonds linguistique mutuellement intelligible, ces Mozambicains sont fréquemment montrés du doigt par un imaginaire collectif qui les rend responsables de tous les maux, de la criminalité à la propagation du virus HIV, en passant par l'accusation rituelle d'accaparer des emplois et de peser sur le taux des salaires ${ }^{1}$. Ce n'est pourtant guère dans le Mpumalanga, mais bien davantage dans les townships de la province du Gauteng autour de Johannesburg, que les Mozambicains ont eu à souffrir des exactions. Détail intéressant, qui révèle que ces émeutes eurent un contenu non seulement xénophobe mais proprement raciste: certaines dépêches de presse que l'on put lire sur Internet après le 10 mai indiquaient qu'à Johannesburg, c'était parfois des gens issus du Mpumalanga, toutes origines confondues, qui, sous l'appellation générique de Shangaan, étaient pourchassés comme indésirables - il en fut de même pour les gens du Limpopo, assimilés à des Zimbabwéens.

\footnotetext{
${ }^{1}$ Cet article fait suite à une première enquête effectuée dans la municipalité de Mbombela en avril 2008 dans le cadre du programme MITRANS, un partenariat entre l'Unité de recherches «Migrations et société » (URMIS), l'Institut français d'Afrique du Sud (IFAS) et le Forced migration studies program (FMSP) de l'Université du Witwatersrand. En association avec le Migrant rights monitoring project "Migration and refugees in medium and small towns" du FMSP, l'essentiel de la recherche a porté sur l'accès des minorités aux services public et privé et au marché du travail, sur leurs relations avec les autorités et la population locales, ainsi que sur le respect des droits humains à leur endroit.
} 


\section{Étrangers et migrants en Afrique du Sud : une histoire mal vécue et mal traitée}

Dans la société post-apartheid, la catégorisation des personnes par nationalité, déjà problématique en soi, montre abondamment ses limites, et cela paradoxalement à un moment de l'histoire de la République sud-africaine (RSA) où cette dernière a besoin, pour s'affirmer comme nation moderne, de revendiquer le sacro-saint état de droit qui distingue le Sud-africain de l'étranger. La contradiction n'est pas mince, si l'on se souvient qu'en même temps, le rachat des fautes de l'apartheid passe par une autre affirmation: celle que «nous, Africains, sommes tous frères au nom d'une origine commune ", au nom de quoi toute xénophobie et a fortiori tout racisme sont impensables. Dès 1995 et de façon accélérée après 2000, cette société a vu affluer un nombre important de personnes du même continent, entrant et s'installant anarchiquement, là où tout déplacement était auparavant strictement encadré, pourvu qu'on ne fût pas blanc. En réaction, les déportations, visant surtout les Mozambicains (et maintenant les Zimbabwéens) ont été massives - de l'ordre de 200000 par an. À la périphérie de Johannesburg, le centre de détention, dit Centre de rapatriement de Lindela, concentre toute la gamme du pire : pathologies, mortalité faute de soins, sévices, viols, maltraitance des mineurs. Significativement, dès 1999, la très modérée Commission des droits de l'homme y dénonçait, outre les violences, la détention de personnes en possession de documents d'identification valides (SAHRC, 1999). Cela indique que très tôt, la RSA post-apartheid a eu quelques difficultés à reconnaître les siens et à distinguer les «bons» des «mauvais» étrangers.

Lors des contacts que j'ai pu avoir avec les divers services, tant au niveau de la municipalité de Mbombela qu'à ceux des autorités fédérales et provinciales, et sans oublier les ONG, mon questionnement portait autour de ces deux interrogations, sciemment naïves dans leur formulation : y a-t-il des problèmes particuliers avec les étrangers? Y a-t-il un traitement particulier de leur cas ? À question idiote, réponse idiote, dit l'adage. Sans doute, mais les réponses s'organisaient de façon instructive autour de deux pôles, entre dénégation et sur-affirmation de la différence nationale. Ces deux positions semblaient se répondre et se compléter sans vraiment se contredire. Elles avaient en commun - ce qui n'est pas sans rappeler le cas de certains pays européens - de signifier : "Faisons comme si les migrants n'existaient pas: nous ne les avons pas vus ». Le premier type de réponse était qu'il ne pouvait pas y avoir dans le pays, ni dans l'application de la loi, de problème de discrimination à l'égard des étrangers, car la constitution l'interdisait ${ }^{2}$ : «Votre enquête n'est pas pertinente, puisque la loi nous fait obligation de traiter toutes les personnes sans discrimination. Pour nous, un étranger ou un Sud-Africain, c'est tout pareil ». (Tout au plus des magistrats ont-ils évoqué la nécessité de maintenir en détention des étrangers lors d'une instruction, faute d'avoir de leur

${ }^{2}$ Ce qui, vérification faite, mais sous toute réserve, paraît faux, puisque le critère de la nationalité est absent de la liste des discriminations énoncées par la Constitution de 1996, qui dit: "The state may not unfairly discriminate directly or indirectly against anyone on one or more grounds, including race, gender, sex, pregnancy, marital status, ethnic or social origin, colour, sexual orientation, age, disability, religion, conscience, belief, culture, language and birth. " [Bill of rights, chapter 2] 
part une garantie de représentation.) La deuxième réponse identifiait au contraire une priorité, voire une exclusivité, d'accès des nationaux aux bénéfices dispensés par l'État. Beaucoup d'agents estiment que des étrangers viennent du Mozambique ou du Swaziland pour profiter du welfare sud-africain, en particulier pour se soigner. On insiste alors sur le fait que la RSA n'a pas les moyens d'assurer l'accueil de tous les étrangers. Il est ajouté que, faute de trouver des ressources suffisantes, les étrangers sont nécessairement poussés vers la délinquance, ou sont au contraire les victimes choisies pour toutes sortes d'abus.

Mais, par delà cette divergence, dont on pressent bien le caractère conjoncturel et volatile, une position semble mettre tout le monde d'accord : c'est celle qui vise spécifiquement les sans-papiers (undocumented). Il est rappelé unanimement que ces personnes ne sont pas fondées à rester dans le pays, et que le seul problème est la difficulté des moyens disponibles pour appliquer la loi, qui se résume en la nécessité de les faire sortir. La question de la pertinence de cette loi et de sa conformité aux droits fondamentaux est très peu mise en cause dans les services. Sur ce point, le discours est unanimement rigide, y compris de la part des ONG. Lors des événements de mai 2008 et de la chasse aux étrangers qui s'ensuivit, certains observateurs remarquèrent la discrétion de ces organisations, habituées à alerter l'opinion sur les arrestations abusives et les atteintes aux droits fondamentaux : dès lors qu'il s'agissait de prendre parti pour défendre les migrants, groupe jugé de moins en moins légitime auprès de la population nationale, contre les attaques venues de cette dernière, l'embarras s'est fait sentir ${ }^{3}$. Pour ne rien simplifier, l'Organisation internationale pour les migrations (OIM) déploie des moyens considérables en faveur du rapatriement dit "volontaire » (bien souvent quasi forcé), des migrants indésirables, propageant ainsi, directement ou par le relais d'associations auxquelles elle négocie ses dotations, l'idée que la question migratoire doit être traitée comme celle d'un excédent d'étrangers qu'il convient de rapatrier.

En résumé, dans l'imaginaire dominant, tout se passe comme si l'illégitimité des étrangers irréguliers rejaillissait sur l'ensemble des étrangers ou réputés tels. Dans la pratique toutefois, les institutions concernées restent soucieuses d'un traitement réaliste des dossiers, et les agents publics comme les activistes oscillent entre les déclarations radicalement hostiles à la reconnaissance de ces undocumented et l'aide pratique qu'ils leur apportent quand il le faut. Aussi, concrètement, chaque agent public, chaque activiste, se forge sa propre doctrine en fonction des situations à traiter : tantôt l'on nous affirmait que, pour secourir quelqu'un en détresse, on ne va pas lui demander ses papiers, tantôt l'on nous disait qu'il fallait bien faire un tri ${ }^{4}$.

\footnotetext{
${ }^{3}$ Aurelia Wa Kabwe-Segatti, 31 octobre 2008 (communication personnelle). Cf. aussi, de la même (26 août 2008), "A New Twist to the South African Migration Debate », <http://sacsis. org.za/site $/$ News $/$ detail.asp?iData $=158 \& i C a t=1441 \& i$ Channel $=1 \&$ nChannel $=$ News\&print $=1>$.

${ }^{4}$ Le «nous » vise ici et plus bas l'assistant de recherche, Vusi Ndukuya, qui m’a été affecté par le FMSP (cf. supra, note 1) et moi-même.
} 


\section{Les migrants « mozambicains " du Mpumalanga, si peu visibles mais si exposés}

Dans un article récent consacré, précisément, aux Mozambicains de Johannesburg, Dominique Vidal observe qu'ils constituent en quelque sorte un "groupe ethnique en creux » : faiblement visibles et peu soucieux de le devenir, relativement dispersés, ne constituant pas une communauté d'intérêts par rapport à tel ou tel type d'activité ou d'intégration, ils contrastent avec les membres d'autres minorités, à l'identité plus affirmée (Vidal, 2008). Un aperçu rapide de leur situation dans la région comprise entre Nelspruit (municipalité de Mbombela ${ }^{5}$ ), capitale du Mpumalanga, et la frontière mozambicaine, donne a priori un tableau très semblable, à cette différence près qu'ils sont donnés par nos interlocuteurs comme omniprésents (surtout quand il s'agit de faire la liste des griefs contre eux), à tel point que souvent le mot «mozambicain» est utilisé comme synonyme de «étranger ", voire de «délinquant» - j'avais noté en 2000 un phénomène semblable à Gênes, ville où le terme marocchino peut signifier alternativement « marocain », «italien du sud» et «bandit». Le caractère fantasmatique de tels amalgames, propagés par la presse à sensation et par la rumeur, est dénoncé par de rares études, qui soulignent que les migrants ainsi visés sont eux-mêmes les premières victimes de cette criminalité qui leur est hâtivement attribuée (Vigneswaran 2008), quand même celle-ci n'est pas exercée à leur encontre par les employeurs ou par les propres forces de police (CRMSA 2007, Wa Kabwe Segatti 2008).

Il reste que, d'après les services compétents, une grande majorité de ces «Mozambicains » sont undocumented, c'est-à-dire sans permis de séjourner, et qu'à ce titre (si l'on peut dire) leur présence sur le territoire est indéfendable. Plus précisément, il s'agit de personnes qui ne possèdent pas le très convoité Identity document (ID), accordé aux citoyens sud-africains et aux immigrants étrangers disposant d'un permis de résidence permanent. Pêle-mêle, ces étrangers sont titulaires de permis de séjour de courte durée, soit valides, soit falsifiés ou obtenus par complaisance, ou bien encore ils sont totalement clandestins vis-à-vis de la loi. Et cela, nous l'avons dit, la société sud-africaine ne peut pas le tolérer, du moins en mots et actuellement.

Aussi, sur le plan des pratiques administratives et policières, leur relative invisibilité est-elle minée constamment, du moins en milieu urbain et périurbain, par leur précarité statutaire. À l'occasion des contrôles dans les chantiers de construction ou dans les zones d'activités artisanales et commerciales informelles, dans les gares routières, lors des roadblocks (barrages routiers) sur la nationale 4 qui mène de Maputo à Pretoria via Nelspruit, ou enfin lors des multiples incidents qui émaillent la vie de cités marquées par la violence, ils ont à faire l'expérience de l'arrestation et de la déportation. La corruption aidant, les volumes des expulsions et des retours s'équilibrent. Comme le disent les personnes qui ont eu à subir des déportations, "si vous avez un billet de banque, vous revenez immédiatement".

Ainsi, la pratique du border hopping (saute-frontière) est devenue un sport, à tel point qu'on m'a raconté le cas d'un expulsé qui avait demandé au chauffeur du

\footnotetext{
${ }^{5}$ Mbombela, ancien nom de la ville de Nelspruit, est aujourd'hui celui d'une communauté de communes urbaines, périurbaines et rurales qui, sur le plan territorial, forme un sous-district.
} 
véhicule qui le reconduisait la possibilité de déposer son bagage en attente chez un ami du côté sud-africain de la frontière. Le border hopping est important pour deux autres raisons : la première est que, du côté swazilandais, les frontières ont été remodelées de telle sorte qu'elles traversent artificiellement des zones socialement intégrées (dans certains villages au sud du corridor de Nkomazi, on passe la frontière le dimanche pour se rendre à l'office religieux); la seconde est que le commerce transfrontalier, de manière analogue, ignore les tracés sur la carte. Mais on peut croire que ce jeu permanent du chat et de la souris, si la frontière peut devenir pour certains une ressource et un business, est pour la majorité des candidats au passage, un rituel ruineux, aussi bien financièrement que pour la santé. Dans le corridor, et jusqu'à Nelspruit, on n'est jamais sûr d'arriver à destination sans s'être fait dépouiller de ce que l'on emmène avec soi, et qui souvent est supposé garantir la dépense du voyageur.

Les services du département des Home Affairs et ceux de la police ont respectivement pour attribution d'expertiser les titres de séjour ou éventuellement d'en fournir si la personne peut justifier d'un emploi légal, et de procéder aux contrôles puis d'expulser le cas échéant. Cette division des tâches, qui n'existe pas dans un pays comme la France, amène un certain pragmatisme dans une coopération qui, lors de mon passage, était réputée s'effectuer dans la bonne entente, contrairement au cas d'autres provinces. Conscients que, face à la situation désastreuse de l'économie mozambicaine, leurs velléités de stopper les flux «clandestins » sont vaines, tantôt ces services ferment les yeux, tantôt ils font preuve de la plus grande brutalité. De surcroît, les autorités locales constatent que le Mozambique est devenu aussi un lieu de transit pour des migrants et des réfugiés venus d'Afrique centrale et orientale et d'Asie. Pour les plus démunis, ces autorités n'ignorent pas non plus que les fermes de cette région, où l'agriculture est prospère mais peu industrialisée, sont gourmandes en main-d'œuvre et absorbent, à l'instar des mines autrefois, une part non négligeable de ces bras venus du Mozambique. Là, loin des regards, ouvriers agricoles, métayers et squatters sont maintenus dans un quasi-servage, avec un ensemble d'exactions (viols, sévices physiques, séquestration, exploitation des enfants) qui retient l'attention d'associations de défense de droits de la personnes, dont les enquêtes, menées en collaboration avec des universitaires, donnent le constat accablant (HRW 2007). Ils n'en sortent parfois que livrés à la police par des fermiers pressés de s'en débarrasser - mais on dit et l'on répète partout que ces fermes constituent des zones de non-droit où les intrusions de la force publique restent exceptionnelles.

\section{Tom : un sans-papiers sous haute protection}

Le parcours de Tom illustre la complexité de certaines situations. Voici quelques éléments de son récit ${ }^{6}$. Son cas, comme on va voir, a fait le tour de la ville, et en quelques jours, ce mois d'avril 2008, nous le croiserons fortuitement dans

\footnotetext{
${ }^{6}$ On ne s'intéresse pas ici à la réalité de tel ou tel élément de ce récit, qui doit être pris pour la vérité de Tom et non pour la vérité en soi. Cependant, vu l'étonnante notoriété de son histoire, tous les faits survenus à Nelspruit nous ont été abondamment confirmés par d'autres interlocuteurs.
} 
quatre lieux différents: la première fois, c'est en bas de l'immeuble qui abrite le consulat du Mozambique, mais c'est un hasard car il sort de chez une avocate spécialisée dans les droits de l'homme. Tom s'appelle peut-être António, et il est né à Nyamatanda, au nord-ouest de Beira, non loin de la frontière zimbabwéenne. Il est mozambicain (de fait, il parle portugais, quoiqu'il préfère l'anglais) mais, confié très jeune à sa grand-mère qui vivait à Harare, il n'a jamais rien eu pour prouver son identité. Il est né en 1971, pense-t-il, auquel cas il fait nettement plus que son âge. De son parcours assez chaotique, on retiendra qu'il a eu plusieurs patrons au Zimbabwe à partir de l'âge de 12 ans, d'abord dans une ferme puis dans le bâtiment (il suivra son chef d'équipe pour un chantier au Mozambique) puis comme vendeur de bananes à Harare, qu'il a essayé de se scolariser, qu'il a eu des démêlés avec la police et qu'en 1993 il décide de quitter le Zimbabwe, où il laisse sa femme et leurs trois enfants. Tom précise qu'entre les deux pays, il n'est pas nécessaire d'avoir de passeport. De retour au Mozambique, il travaille à Gorongoza (province de Beira), prend une autre femme et a deux autres enfants. En 2001, il suit son employeur dans la capitale.

Là, à Maputo, c'est en 2005 qu'il rencontre un Afrikaner de Nelspruit, qui lui propose de venir le rejoindre en Afrique du Sud, à Mbombela. Tom part voir sa femme, qui tente de le dissuader, mais le patron lui promet 3000 rands $^{7}$ par mois et lui propose une avance de 1500 rands, à la suite de quoi il finit par se décider. Comme convenu, un jour il téléphone de la frontière, où il est bloqué, n'ayant ni passeport ni argent. À deux heures du matin, un chauffeur afrikaner vient le prendre et paye la «taxe» pour lui. Nous sommes à la fin de l'été austral. Sur un chantier éloigné de la ville, il pose des carreaux - «cela, je savais faire $»^{8}$. Au bout d'un mois, il n'a rien reçu, sauf 50 rands concédés par la femme du patron. Il lui est expliqué qu'il n'a pas besoin d'argent, puisqu'on pourvoit à tout et qu'il n'a pas sa femme avec lui. S'il a besoin de quelque chose, il suffit qu'il demande. Deux autres mois passent, et il se décide à demander son salaire au foreman (chef d'équipe ou bras droit du patron, selon la taille de l'entreprise), qui lui répète ceci : «Tu vas d'abord dans ton pays, et là tu recevras. Je ne peux pas te donner ton salaire ici. Va chercher ton sac et nous irons voir le patron ». Là, Tom comprend trop tard qu'il est tombé dans un piège : le patron se saisit d'un gros bâton, le bastonne d'importance en cherchant surtout à viser le dos, et le menace de ses chiens s'il tente quoi que ce soit. De retour dans sa chambre, il constate que son compagnon de chantier refuse de l'aider à s'enfuir par peur des représailles. Il finit tout de même par déjouer la surveillance des vigiles, s'enfuit et va se réfugier à la police de Nelspruit (rappelons que Tom n'a aucun document), qui l'emmène sans attendre à l'hôpital. C'était le 17 juillet 2005. Là, survient le patron avec deux «collègues » et deux chiens : "Comment es-tu arrivé ici ? - C'est le gouvernement - Quel gouvernement? - La police ». Le patron cherche à le récupérer, mais en vain, part à la police, où il explique que Tom est un affabulateur, qu'il n'est pas mozambicain mais zimbabwéen.

\footnotetext{
7 Environ 300 euros en 2005, 240 euros en 2008.

8 Tiles en anglais. Désirant savoir s'il s'agissait de tuiles ou de carreaux, j’ai posé la question en portugais, et j'ai eu la surprise de voir que Tom ne comprenait pas le mot tïolo.
} 
C'est ainsi que, quand nous le rencontrons, Tom est là depuis presque trois ans. Vivotant entre Nelspruit et son compound (habitat rural isolé adossé à une ferme) à une quinzaine de kilomètres, il est sous la triple protection de la police, qui sursoit à la déportation tant que l'affaire n'est pas passée en justice, du département du Travail qui a tenté de convaincre son patron de lui signer un contrat de travail, et des Home Affairs, qui attendent un contrat pour lui procurer un permis de séjour. Un intermédiaire mozambicain spécialisé dans le placement de ses compatriotes se faisait fort d'obtenir 1000 rands mais, son patron lui en devant 7 500, Tom a refusé. La suite est plus embrouillée car Tom oriente son récit pour nous convaincre de mettre la main à la poche. En effet, il est dégoûté, et son plus cher désir serait de repartir et de rejoindre sa famille. Mais le voyage est coûteux et il ne peut pas non plus arriver les mains vides. Bref, quand il aura rassemblé 9000 rands, il partira, mais il est loin du compte. Plus tard, un des services s'occupant de son cas nous suggérera qu'il n'est sans doute pas pressé de repartir car il a déjà reçu un pécule l'an dernier, qu'il a immédiatement dépensé en achetant TV, chaîne HiFi, etc. En attendant, la police lui a fourni un gilet fluo et confié la garde du parking d'un petit centre commercial.

Avant tout, retenons de ce récit l'idée que, avec ou sans souffrance (mais sans doute plus souvent avec), la condition de migrant est en soi un métier, un métier qui s'apprend à travers des vicissitudes, et que caractérise une permanente incertitude, aussi bien quant aux ressources que l'on trouvera que quant aux relations sociales qu'on pourra lier. Pour les aventuriers et les ouvriers ou commerçants itinérants, le parcours s'apparente à un jeu de l'oie: on progresse, on se fait dépouiller, on repart en arrière, on reconstitue son épargne ou bien on dépense tout, on se crée une sociabilité ou on s'isole, et tout ce système complexe de paramètres, à son tour, renforce la mobilité ou l'affaiblit.

\section{Qu'est-ce qu'un Mozambicain en RSA ?}

Mais surtout, par rapport à notre propos, qui est d'interroger le classement par nationalité, le témoignage ci-dessus a l'intérêt de montrer la persistance des rapports sociaux de race et de nationalité, avec leur brutalité typique, telle qu'ils œuvrent dans ce bastion afrikaner qu'est le Lowveld. Ce qui a dû provoquer la ruine de Tom, c'est qu'il avait une bonne qualification et de l'ambition. Mais le sort d'autres ouvriers migrants est plus indifférencié, et quotidiennement plus cruel peut-être. À Nelspruit, centre administratif et principale place commerciale de la province, le visiteur peut voir la maraude quotidienne de ces énormes pick-up, où dans la cabine on aperçoit le foreman assis à côté du maître, qui sillonnent le centre de la ville à la recherche de journaliers et de tâcherons postés aux carrefours, qu'ils font grimper à la hâte sur la plate-forme arrière. Le nombre de candidats est tel qu'on imagine les prétentions financières de l'ouvrier se réduire à un "votre tarif sera le mien ». Il s'agit probablement de ces fameux Mozambicains et autres étrangers, invisibles mais si présents. Car, du côté patronal, on ne manque pas de répéter que les travailleurs locaux ne valent rien et que les Sud-Africains sont paresseux et exigeants : mille préférences pour ces migrants peu exigeants, réputés durs à la tâche et techniquement performants, et qui ignorent, espère-t-on, le sens du mot «contrat ». Le soir à la brume, il arrive qu'on croise à nouveau les mêmes 
pick-up sur les parkings des agences bancaires, où le personnel de la journée attend, gris de poussière, le retour du maître avec les billets de la paye. Puis soudainement, la nuit venue, la ville devient déserte, et le même visiteur se demande où est passé tout ce monde - on y reviendra.

Notre ami Tom est-il mozambicain ? Son patron en doutait, et de toute manière, il n'a autre aucun document que le sauf-conduit que lui ont délivré les Home Affairs. La population «mozambicaine » du Mpumalanga est le produit composite d'une série de peuplements ou de passages successifs, aujourd'hui plus ou moins consolidés et vecteurs ou supports d'autres mouvements, qui viendront à leur tour remodeler la configuration d'ensemble jusqu'à rendre inextricable le tableau général et inintelligibles les situations individuelles. Dans cet entremêlement interviennent notamment les questions du statut légal, du travail et de l'habitat.

La population de Mbombela est majoritairement composée de Noirs (93\% au recensement de 2001). Les langues parlées sont le siSwati (89\%), l'afrikaans et l'anglais. Il n'existe pas de statistiques fiables concernant la place de la population étrangère dans le total, et la municipalité ne paraît pas impatiente de dénombrer les étrangers, ce qui pourrait être perçu comme une reconnaissance de la légitimité de leur présence comme tels. De la sorte, les habitants étrangers et nationaux supportent ensemble le fardeau de la réputation de la zone où ils habitent. À côté des Mozambicains, les principaux groupes concernés sont les Swazis et, depuis quelques années, les Zimbabwéens. Mais on rencontre également à Nelspruit diverses nationalités telles que Kényans, Pakistanais, Somalis, notamment dans le commerce de gros et demi-gros.

Le cas des Mozambicains, qui sont les plus nombreux dans le Lowveld, illustre la difficulté qu'il y a à définir rigoureusement le statut selon les deux classifications de base, qui sont respectivement: citoyens sud-africains / étrangers autorisés (critère de la nationalité) et étrangers autorisés / étrangers undocumented (critère du titre de séjour). La nationalité sud-africaine elle-même est un cas problématique. La guerre civile au Mozambique, par ailleurs largement attisée par la RSA, a provoqué l'irruption de centaines de milliers de réfugiés, qui venaient s'ajouter aux mineurs sous contrat, recrutés de longue date en vertu d'accords entre les deux pays. Ces réfugiés, à la suite d'un terrible parcours du combattant (traversée du parc animalier, franchissement d'une barrière sous haute tension), n'étaient tolérés par le gouvernement sud-africain que s'ils demeuraient dans les homelands (bantoustans) de Gazankulu et KaNgwane, éparpillés et mis sous administration tribale en vertu du système d'apartheid, où ils étaient une proie facile pour les fermiers de la province. En 1990, leur nombre était estimé à 350000 . Non reconnus, ils ne possédaient aucun document de voyage et ne pouvaient pas bénéficier de la protection du HCR. La guerre civile prit fin en 1992, précisément au moment où l'apartheid tombait en lambeaux et où le pays s'apprêtait à réintégrer les territoires "autonomes » dans la nation sud-africaine. Mais, même après l'abolition de ces homelands en 1994, ils ne reçurent aucun document d'identité, ce qui revenait à empêcher leur circulation hors de ces territoires (HRW 1998 : 29-31 - et même, à la limite, à y rendre leur présence illégale.

Ainsi, les identités, l'ancienneté, la localisation même des personnes n'ont jamais constitué un cadre assuré pour appliquer clairement des règles de nationalité et de séjour. Certains Mozambicains furent rapatriés «volontairement » dès 1993 
sous les bons offices du HCR, tandis que d'autres, à condition d'avoir fui leur pays entre 1985 et 1992, obtenaient le titre de réfugiés prima facie (c'est-à-dire indistinctement, au vu d'une origine commune) et que d'autres encore, n'étant éligibles à aucune de ces deux catégories, étaient jetés dans l'illégalité. Les travailleurs mozambicains sous contrat furent aussi parmi les victimes de ces arrangements, puisque la fin des bantoustans en faisait subitement des indésirables, sinon des illégaux dans le territoire réunifié. De leur côté, au nom du fait que seul à terme le rapatriement était la solution, les réfugiés provisoirement reconnus comme tels étaient mis en position de totale précarité, n’ayant pas même accès de plein droit au marché du travail. Destinées sans succès à apurer la situation, des amnisties furent proclamées, notamment en 1996 et en 1999, mais sont restées dérisoires proportionnellement aux expulsions en masse dont chacun s'accorde à admettre l'inefficacité. Plus encore peut-être que le régime ancien, la sortie de l'apartheid s'est présentée, dans cette province, comme une implacable machine à fabriquer l'illégalité de ses migrants et à en empêcher l'intégration, quel qu'en soit l'enracinement dans la société locale ${ }^{9}$.

\section{Travailler, se déplacer, se loger : nouvelles formes de la discrimination}

Les migrations transfrontalières, qui étaient de tradition, n’ont jamais cessé dans le Mpumalanga, où elles restent alimentées par un important différentiel d'emploi et de richesse. Aux personnes évoquées ci-dessus, victimes d'un changement des règles du jeu constitutionnel, se sont ajoutées classiquement ensuite celles qui sont entrées sans permis ou celles qui ont dépassé la durée de séjour autorisée. Pour faire face à la pression à la frontière mozambicaine et pour ne pas empêcher l'approvisionnement en main-d'œuvre de certains secteurs, le gouvernement provincial s'est résolu à apporter des aménagements au contrôle des entrées, certes minimes, mais supposés éviter que le situation soit ingérable. Ce dispositif légal s'additionne à la corruption aux points de passage pour maintenir une certaine porosité de la frontière. Depuis 2005, des visas de trente jours sont délivrés, qui facilitent les activités commerciales, très intenses entre Maputo et Nelspruit, tout en contribuant, de l'avis des autorités compétentes, à l'augmentation du stock d'irréguliers dans la région.

En outre, dans la province du Mpumalanga, un accord de main-d'œuvre passé avec la province de Maputo a mis en place un système de cooperate permits, calqué sur l'ancien statut des travailleurs des mines. Par ce biais, dans les exploitations agricoles et forestières, des employeurs sont invités à faire des demandes de recrutement en fonction de leurs besoins auprès d'une agence de placement mozambicaine sise à Nelspruit, pour des contrats de douze mois renouvelables. Les travailleurs ainsi mobilisés, sous réserve d'une «lettre de non-objection » délivrée par les Home Affairs, bénéficient d'un certificat d'embauche - la régularité de leur séjour étant liée à leur contrat particulier. Quant aux employeurs ruraux, une fois loin des regards de l'inspection du travail, ils sont suspectés de ne pas accom-

\footnotetext{
9 Pour la période de transition jusqu'en 2001, voir l'analyse détaillée et pénétrante proposée par Aurelia Wa Kabwe-Segatti (2002). Cf. aussi Polzer (2005).
} 
plir leurs engagements en termes de salaire ou de conditions de logement ${ }^{10}$. Outre les cooperate permits, la loi sur le travail autorise la signature de contrats et la régularisation des cas à condition que les possibilités offertes par le marché de l'emploi national aient été explorées préalablement. Vu la nature du peuplement telle qu'on l'a évoquée, ce mode de recrutement ne peut être que très marginal, puisque toute personne qui se présente aux Home Affairs avec un contrat d'embauche risque la déportation pour défaut de permis de séjour - en outre, les dossiers sont très lourds à constituer.

Quoiqu'il n'existe pas encore d'étude approfondie sur la place des Mozambicains sans permis dans le marché du travail - la seule chose assez certaine étant qu'ils constituent la grande majorité de leurs compatriotes-, les témoignages des personnes à qui leur pratique apporte une connaissance partielle de leur situation convergent pour donner le profil suivant. Une grande partie d'entre eux sont employés dans les fermes, mais subissent un turnover important en raison du caractère saisonnier des emplois et à cause des conditions très dures qui y règnent. On a parlé plus haut des migrants qui s'embauchent à la journée pour des tâches aux alentours de la capitale. Quant aux travailleurs mozambicains en milieu urbain, leurs qualifications sont en général supérieures à celle des nationaux mais ne sont pas reconnues au niveau du salaire ou de la promotion. Face à cette situation, les syndicats, qui se plaignent de la méconnaissance de leurs propres droits, se plaignent des pratiques de dumping social qui instrumentalisent l'irrégularité de leur situation. Indépendamment du secteur de la construction, très actif, et de celui de la sécurité, les Mozambicains qualifiés en mécanique automobile, plomberie, électricité etc. sont recherchés, si bien que, à l'instar de ce qui arriva à Tom le carreleur, les employeurs vont les rechercher au Mozambique. À Nelspruit, on en trouve également beaucoup comme employés de commerce dans les établissements du centre, dans les activités de rue et comme vendeurs ambulants (hawkers). Ce sont plus particulièrement les femmes qui semblent vouées aux activités commerciales de type pendulaire ente les provinces frontalières, une partie de leurs marchandises étant utilisée pour payer le transport. D’après quelques témoignages, comme il a été dit, la migration n'est pas nécessairement tracée d'avance : si les biens ont été mal vendus ou volés ou confisqués, si les affaires ont été maussades, l'itinéraire s'arrêtera à Mbombela, voire à Nkomazi, première municipalité à l'est de la frontière. Dans le cas contraire, la commerçante pourra poursuivre sa route vers Pretoria ou Johannesburg. Cette indécision des destinées est à mettre en relation avec l'incertitude des statuts telle qu'elle a été résumée plus haut.

Un élément particulièrement important dans la détermination de ces trajectoires est celui de l'insécurité, ce qui nous amène à conclure sur la question du transport, qui renvoie à son tour à celle, cruciale entre toutes, du logement. C'est à plusieurs titres en effet que, malgré leurs protestations de citoyenneté (Polzer 2004), les Mozambicains se voient rappeler sans répit qu'ils ne sont pas chez eux.

${ }^{10}$ Il ne m'a pas été possible d'en savoir plus sur le fonctionnement de ces cooperate permits, juste assez toutefois pour comprendre que, d'une part, ce système semblait dépourvu de transparence et, d'autre part, le volume de main-d'œuvre concerné était minime par rapport au nombre total de travailleurs mozambicains employés dans les fermes. 
À Nelspruit, que ce soit au marché, dans les boutiques, à la gare routière ou dans les rues, ils travaillent en tout lieu. Cette capitale abrite les administrations de tous les échelons territoriaux. Située au cœur d'une riche région minière et agricole, ancienne station du chemin de fer Pretoria-Lourenço Marques (devenu Maputo), elle doit à sa position géographique d'être aujourd'hui un important point de passage des échanges routiers de voyageurs et de marchandises avec ces deux pays voisins, le Mozambique et le Swaziland, dont la bourgeoisie vient faire ses achats dans les luxueux centres commerciaux. Les entrepôts, les concessionnaires automobiles y prolifèrent. Le secteur de la construction connaît une forte croissance. En prévision de la Coupe du monde de 2010, un stade de 45000 places est en cours d'achèvement à l'ouest de la cité (où des arrangements ont amené à l'embauche prioritaire d'ouvriers nationaux et locaux). Très étendue, la ville est peu verticalisée, à l'exception du centre: la densité de population est faible, l'habitat est de type pavillonnaire dominant, et de nombreuses friches sont l'objet d'une intense spéculation. En l'absence de réserves foncières qui auraient permis à la municipalité d'enrayer la hausse du prix des terrains constructibles, les loyers en milieu urbain sont de plus en plus élevés, et de toute façon inaccessibles à la plupart, y compris les classes moyennes: en réalité, seule une petite proportion de la population de la municipalité habite à l'intérieur des limites territoriales de la ville de Nelspruit qui, une fois la nuit tombée, devient une ville morte. Il en va de même des deux autres communes, Hazyview et White River, qui constituent ensemble, avec les diverses zones périurbaines et rurales environnantes, la municipalité de Mbombela.

La question du logement à Mbombela illustre le poids des structures urbaines héritées de l'apartheid. Une stratification spatiale complexe s'est effectuée au cours de la période de transition, sur la base des frontières héritées de l'apartheid, qui se sont largement reproduites dans la pratique tout en étant officiellement dénoncées par la nouvelle constitution. La ségrégation urbaine continue de frapper l'ensemble de la population laborieuse pauvre. Fondamentalement, la division spatiale reflète une division en classes sociales, qui se superpose à une stratification raciale ou nationale de la population. Grossièrement, on peut distinguer quatre ensembles de strates.

Le premier ensemble est constitué par les villes : Nelspruit par exemple. Elles fournissent beaucoup d'emplois formels et informels et concentrent la plupart des services et administrations; mais elles offrent, on l'a vu, peu d'opportunités de logement à la population pauvre. Globalement, seuls les Blancs et quelques riches Indians et Coloured ont des titres de propriété (title deeds). Les extensions à la périphérie ont souvent répondu aux stratégies de ségrégation destinées à écarter certains groupes du centre ville : ainsi, à l'est de Nelspruit, on a fondé les quartiers de Valencia pour les Indians et Nelsville pour les Coloured, avec aussi aujourd'hui un pourcentage notable de sans-abris (homeless). On trouve enfin à Nelspruit quelques dizaines de lits pour homeless gérés par une ONG chrétienne et des taudis (shacks) de petite taille aux entrées de la ville.

Les deux ensembles suivants résultent de la politique de déportation (removal) mise en place par l'apartheid. Dès les années 1960, les habitants de Nelspruit (alors nommée Mbombela) furent déplacés à proximité des fermes de la région, puis installés dans les années 1970 dans les townships de KaNyamazane, Matsulu 
et Kabokweni à l'est, ainsi que Bushbackbridge à la frontière avec le Limpopo. Par la suite, avec la densification de l'habitat, une nouvelle déportation (removal) de Noirs eut lieu vers des réinstallations (settlements) situées dans la juridiction des homelands Gazankulu et surtout KaNgwane (dont le territoire se partage aujourd'hui entre les municipalités de Mbombela et Nkomazi, à la frontière avec le Swaziland et le Mozambique), comme Pienar - il y eut ensuite des mouvements de retour est-ouest, les personnes cherchant à se rapprocher de l'aire d'influence de Nelspruit. Ces deux types de peuplement correspondent respectivement aux statuts fonciers (land tenure) R293 et R188. Aujourd'hui, les townships sous R293 sont généralement soumis au régime du deed of grants (concession du terrain sans propriété); on $\mathrm{y}$ trouve un minimum de services et d'infrastructures, dont l'alimentation en eau et la viabilisation des voies d'accès. Après l'extinction des homelands, les settlements sous R188 sont demeurés sous administration tribale, une faible redevance annuelle étant perçue par le chef traditionnel en échange d'un PTO (permission to occupy); parfois immenses et situées loin des axes routiers, ces zones sont beaucoup moins fournies en services et en infrastructures; la criminalité y est un des sujets les plus commentés par la population. On aura remarqué que les zones héritées de l'apartheid sont précisément celles où furent assignés à résidence les réfugiés mozambicains de la guerre civile. Par la suite, les nouveaux migrants mozambicains, soucieux de se rapprocher des opportunités informelles d'emploi, tentent d'y solliciter une concession : officiellement, en tant qu'étrangers illégaux (illegal aliens), ils n'y ont pas droit, mais ils trouvent notoirement place dans les interstices de cet habitat périurbain, surtout sous R188, qui connaît une densification certaine.

Le dernier ensemble est constitué des informal settlements alimentés par la pression démographique et migratoire intérieure et extérieure, ainsi que par les retards pris dans la politique de réintégration et les expulsions des fermes : occupations illégales des terres municipales dans les townships, peuplement des terres d'État, notamment sur les bords de route, compounds aux abords des fermes. Cet habitat contribue à l'urbanisation des zones rurales hors de toute planification des infrastructures avec, comme en tout lieu, une spéculation sur les droits d'usage.

Ce schéma stratifié constitue l'héritage actuel : en fonction de leur revenu, de leur catégorie raciale et de leur statut (nationaux, migrants, réfugiés), les travailleurs sont contraints de s'installer dans des zones plus ou moins éloignées de la capitale Nelspruit, avec les possibilités offertes par celle-ci en matière d'emploi et d'accès aux services. Le confort de l'accès aux ressources de la ville devient alors le témoin et le vecteur d'une ségrégation sans cesse remodelée, où, à bien des égards, les «Mozambicains» (ce qui inclura les autres étrangers de statut précaire) ont pris la place des blacks de l'apartheid.

Dès lors, la possibilité de se déplacer sans danger devient l'alpha et l'omega des stratégies quotidiennes. Toute la population est concernée par le risque de l'agression qui, par sa dimension obsédante et soigneusement entretenue par les agences qui opèrent sur le marché de la sécurité privée, ponctue l'horaire des travailleurs. Dès quinze heures, il devient difficile d'être reçu dans un service administratif et, dès dix-sept heures, les abords de la gare routière offrent le spectacle hallucinant d'un sprint collectif pour attraper les bus. Cette ségrégation spatiale n'est propre ni à la zone concernée ni à l'Afrique du Sud. Mais elle 
reproduit abondamment les stratifications (sociales, raciales ou nationales), non seulement comme en tout lieu par ses effets sur la durée réelle des journées, mais par l'inégalité qu'elle crée devant le risque criminel. Les plus vulnérables sont ceux qui restent les derniers, qui n'ont pas de moyen de transport assuré, et qui doivent rejoindre les points les plus éloignés des axes de circulation. Aussi n'est-ce pas la moindre des surprises pour le visiteur européen que d'apprendre que les zones rurales sont considérées comme les plus exposées aux crimes. La question particulière des étrangers ne peut pas être comprise sans prendre en considération ce cadre, dans la mesure où la précarité de leur insertion rend, plus encore que pour les nationaux, vitale la question de leurs déplacements. Pour le moment, le problème de l'insécurité est envisagé exclusivement sous les angles de la menace préventive et de l'intervention répressive, alors que le risque criminel est inscrit dans la structure même de la ségrégation spatiale et des incertitudes qu'elle produit. La stabilisation des étrangers (dans la mesure bien sûr où elle est souhaitée par ces derniers) est un défi majeur de la société sud-africaine dans sa volonté de sortir ses pas des mauvaises empreintes de l'apartheid.

23 janvier 2009

Alain MORICE

Université Paris Diderot

Unité de recherches «Migrations et société »(URMIS) morice@univ-paris-diderot.fr

\section{Références}

Consortium for Refugees and Migrants in South Africa (CRMSA) 2007, Protecting Refugees and Asylum Seekers in South Africa, Pretoria, CRMSA, $56 \mathrm{p.}$

Human Rights Watch (HRW), 1998, "Prohibited Persons". Abuse of Undocumented Migrants, Asylum Seekers and Refugees in South Africa, New York, HRW, $236 \mathrm{p}$.

— 2007, "Keep Your Head Down". Unprotected Migrants in South Africa, New York, HRW Reports, vol. $19(3 \mathrm{~A}), 112 \mathrm{p}$.

Polzer, T. 2004, "We are all South Africans now" : the Integration of Mozambican Refugees in Rural South Africa », Johannesburg, FMSP (University of the Witwatersrand), 10 p, («FMSP Working Paper Series », 8).

— 2005, Adapting to Changing Legal Frameworks: Mozambican Refugees in South Africa, Johannesburg, FMSP, 29 p. («FMSP Working Paper Series », 17).

South African Human Rights Commission (SAHRC) 1999, Illegal? Report into the Arrest and Detention of Suspected Undocumented Migrants, Johannesburg, $56 \mathrm{p}$.

VIDAL, D. 2008, "Vivre sur fond de frontières. Les migrants du Mozambique à Johannesburg ", Cultures \& Conflits, 72: 101-107.

Vigneswaran, D. 2008, «Undocumented migration : risks and myths (1998-2005)», in A. Wa Kabwe-Segatti (ed.), Migration in post-apartheid South Africa. Challenges and questions to policy-makers, Paris, Agence française de développement (AFD) : 135-162.

Wa Kabwe-Segatti, A. 2002, «Du rapatriement volontaire au refoulement dissimulé. Les réfugiés mozambicains en Afrique du Sud», Politique africaine, 85, mars : 75-92.

— ed. 2008, Migration in post-apartheid South Africa. Challenges and questions to policy-makers, Paris, AFD, $238 \mathrm{p}$. 